\title{
COMPTE RENDU D'UNE PREMIĖRE REPRODUCTION EN FRANCE DE L'ESTURGEON SIBÉRIEN ACIPENSER BAERI
}

Patrick WILLIOT, Thierry ROUAULT (1)

\section{RÉSUMÉ}

Cette reproduction a été faite avec 5 femelles et 7 mâles sélectionnés parmi un lot d'animaux importés d'U.R.S.S.

Les femelles pesaient environ $5 \mathrm{~kg}$, les mâles $3 \mathrm{~kg}$.

L'ovulation a été obtenue sur les 5 femelles quel que soit le traitement hypophysaire (hypophyse d'esturgeon et de carpe).

La spermiation a été obtenue sur seulement 3 mâles, l'injection d'hormone gonadotropine chorionique (H.C.G.) n'ayant donné aucun résultat.

Pour éviter que les œufs ne se collent, plusieurs traitements ont été testés : une solution d'acide tanique, du lait et une suspension d'argile qui s'est révélée être la meilleure méthode dans nos conditions expérimentales.

L'incubation a eu lieu en bouteille de Zoug.

Les premières éclosions sont intervenues après environ $160^{\circ} \mathrm{C}$ jour. Le nombre de larves obtenu a été estimé à $9000 \pm 1000$.

\section{SUMMARY}

This reproduction was achieved with 5 females and 7 males chosen among animals imported from U.S.S.R.

The females weighed $5 \cdot \mathrm{kg} \pm 0,9$, the males $3 \mathrm{~kg} \pm 0,5$.

Whatever the pituitary treatment was (pituitary of sturgeon or carp) ova were obtained from the 5 females.

The malès were also tested with pituitary of sturgeon and carp and had H.C.G. injection. Only 3 males gave sperm and H.C.G. gave no result.

Three treatments were tried to prevent the eggs from sticking to one another : tanic acid, milk and clay suspension, the latter being the best method under our experimental conditions.

Incubation was made in Zug jars. First hatching occured after about $160^{\circ} \mathrm{C} x$ day. The estimated number of larvae obtained was $9000 \pm 1000$.

Cette espèce, très faiblement amphibiotique, est naturellement présente dans les fleuves sibériens.

Les animaux sur lesquels ont été effectués les travaux relatés dans cette note proviennent d'U.R.S.S. Ils ont été importés fin 1975, à titre d'échange, dans le cadre de la coopération franco-soviétique dans le domaine de l'océanologie. Leur poids moyen était d'environ $360 \mathrm{~g}$ avec pour extrêmes 50 et $500 \mathrm{~g}$. Ces animaux sont nés début juillet 1974 .

Alors que les soviétiques ont beaucoup travaillé sur leurs espèces migratrices (Huso huso, Acipenser guldenstädti et Acipenser stellatus) l'esturgeon sibérien Acipenser baeri a fait l'objet jusque là de peu de travaux.

Les techniques de reproduction utilisées par les soviétiques sur les grands migrateurs sont maintenant bien connues (WILLIOT, CHARLON, 1978); nous ne disposons par contre d'aucune information sur les méthodes relatives à l'esturgeon sibérien.

L'intérêt des recherches sur cette espèce est double: acquérir une expérience utilisable au moins en partie sur l'espèce indigène (Acipenser sturio) en voie de disparition, augmenter notre stock très limité, cette espèce pouvant peut-être être intéressante en pisciculture. Pour cela il était nécessaire de tester les techniques connues sur d'autres espèces à l'esturgeon sibérien et de les adapter au contexte français.

(1) Ministère de l'Agriculture, CEMAGREF, Division Aménagements Littoraux et Aquaculture, 50 avenue de Verdun, BP 3, GAZINET, 33610 CESTAS PRINCIPAL 


\section{CONDITIONS D'ÉLEVAGE (BARRUCAND et col. 1978)}

Jusqu'en 1978, les animaux étaient partagés en 2 lots :

- l'un était élevé dans des bassins en béton alimentés par les eaux de refroidissement de la centrale EDF d'Ambès (Gironde). La température de l'eau variait de $14^{\circ} \mathrm{C}$ à $28^{\circ} \mathrm{C}$ avec un pic à $34^{\circ} \mathrm{C}$ durant l'été 1976 entraînant des mortalités notables (C.T.G.R.E.F., juin 1979).

- l'autre se trouvait dans la pisciculture de Donzacq (Landes) gérée par le laboratoire de nutrition des poissons de I'INRA (Centre d'Hydrobiologie de St Pée sur Nivelle).

Cette pisciculture est alimentée par une source dont la température est quasiment constante tout au long de l'année $\left(16\right.$ à $\left.18^{\circ} \mathrm{C}\right)$. Les parois des bassins sont en ciment, le fond en sable.

Depuis fin 1978 tous les poissons ont été regroupés dans cette pisciculture et nourris avec des granulés pour truites.

\section{CONTROLE DE LA MATURATION - SÉlECTION DES GÉNITEURS}

Ces contrôles ont été effectués par biopsie (WILLIOT, CHARLON, 1978). Après anesthésie au phénoxyéthanol $\left(0,5 \mathrm{~cm}^{3} / 1\right.$ pendant environ 5 minutes) la paroi abdominale est incisée.

Lorsque les individus sont jeunes cette technique est utilisée pour sexer les animaux. Dans ce cas, il est souvent nécessaire d'ouvrir sur 4 à $5 \mathrm{~cm}$. La plaie est refermée à l'aide de points de suture.

Dans le cas d'animaux dont les gonades sont déjà bien développées $1 / 2$ à $1 \mathrm{~cm}$ suffisent pour faire un prélèvement et une observation. Souvent il n'est pas nécessaire de recoudre la plaie pour obtenir la cicatrisation.

Les critères d'appréciation de la maturation sont les suivants:

pour les 2 sexes l'aspect général des gonades (développement, graisse)

- pour les femelles: - diamètre, couleur, aspect des ovocytes

- vésicule germinative plus ou moins nette

- pourles mâles: - possibilité d'obtenir du sperme par écrasement d'un petit morceau de gonade.

L'utilisation de ces critères a permis en octobre 1980 de choisir 30 animaux parmi l'ensemble (environ 80 ).

Deux observations doivent être mentionnées:

- le degré de maturation est très variable selon les individus de même sexe

- pour une même femelle, on constatait parfois une grande hétérogénéité dans l'évolution des ovocytes.

Tous les animaux portent une marque ovale de chez PRESADOM sur le premier rayon de la nageoire dorsale.

Dans l'espoir d'obtenir une évolution finale de la gamétogenèse plus homogène, ces 30 poissons ont été transférés le 28 octobre dans un étang de Brenne. La température de l'eau était alors égale à $13^{\circ} \mathrm{C}$.

Le 24 février 1981 , l'étang a été pêché, la température de l'eau était de $4^{\circ} \mathrm{C}$; après un nouveau contrôle de maturation 12 animaux ont été sélectionnés: 5 femelles et 7 mâles.

Tous les animaux avaient maigri au cours de cet hivernage :

Poids en $\mathrm{kg}$

\begin{tabular}{|l|l|l|}
\cline { 2 - 3 } \multicolumn{1}{c|}{} & $27-10-80$ & $24-02-81$ \\
\hline femelles $(n=5)$ & $5,4 \pm 0,89$ & $5,12 \pm 0,87$ \\
\hline mâles $(n=7)$ & $3,37 \pm 0,44$ & $3,07 \pm 0,51$ \\
\hline
\end{tabular}


Jusqu'au 27 février les animaux sont restés dans une eau courante à $4^{\circ} \mathrm{C}$; le 27 , ils ont été placés dans les bassins de l'écloserie (1). En 48 heures la température de l'eau a été augmentée progressivement de $4^{\circ} \mathrm{C}$ à $11^{\circ} \mathrm{C}$ puis est maintenue constante.

\section{REPRODUCTION}

Les Soviétiques utilisent sur leurs grands migrateurs une technique caractérisée par 3 aspects importants:

- une seule injection d'hypopnyse d'esturgeon à raison de $2 \mathrm{mg} / \mathrm{kg}$ pour les femelles

$1 \mathrm{mg} / \mathrm{kg}$ pour les:mâles

- traitement anti agglomérant des cufs par de l'argile

- incubation dans des appareils spécifiques

Certains aspects sont pour l'instant non généralisables en France.

\subsection{Injection d'hormone}

Nous ne disposions que d'une très faible quantité d'hypophyses d'esturgeon (Acipenser guldenstädti) (2). d'autres.

De plus, il était impossible d'envisager à moyen terme la possibilité d'en obtenir

Il était alors nécessaire de tester l'efficacité d'autres sources d'hormone faciles à obtenir ; nous avons choisi d'utiliser:

- des hypophyses de carpe

- de la gonadotropine chorionique humaine (H.C.G.).

D'autre part, sur les femelles de cyprinidés il est courant de partager la dose globale en plusieurs injections (MARCEL, 1980). Cette méthode de fractionnement augmente l'efficacite de l'hormone (BRETON, FOSTIER, JALABERT, WEIL, 1980).

Enfin selon WOYNAROVITCH (com. pers.) la première injection devrait être effectuée avec une hormone spécifique alors que pour les suivantes il serait possible d'utiliser des sources d'hormone plus diversifiées.

Cette hypothèse a donc été retenue. Les doses globales d'hypophyses retenues sont les suivantes:

- hypophyses d'esturgeon (H.e.) $q 2 \mathrm{mg} / \mathrm{kg}, \sigma^{\prime} 1 \mathrm{mg} / \mathrm{kg}$

- hypophyses de carpe (H.c.) Ơ et $q 6 \mathrm{mg} / \mathrm{kg}$

- hormone chorinique gonadotrope (H.c.g.) ơ 1000 U.I./kg

Lorsque 2 sources d'hypophyses ont été utilisées sur un même animal, la dose totale correspond à 5 ou $6 \mathrm{mg} / \mathrm{kg}$.

Le tableau ci-après indique le détail des injections et les observations.

\subsection{Observations}

Les femelles: Les observations étaient effectuées en tenant les femelles verticalement, tête en haut et en pressant les flancs afin d'obtenir quelques ovules par l'orifice génital. Ainsi pour 3 d'entre elles, cela a été observé environ 50 heures après la 1 ère injection. Parmi ces 3 femelles, 2 avaient reçu une seule injection avec de l'hypophyse d'esturgeon, la troisième ayant reçu 2 injections d'hypophyse d'esturgeon et une 3ème injection d'hypophyse de carpe. (1979).

Ce délai de réponse correspond aux données établies par A.A. KHAKIMOULLIN

Pour les 2 autres femelles, la fréquence d'observation trop lâche ne permet pas de préciser le délai de réponse.

Ce que l'on peut constater c'est que l'ovulation a eu lieu quel que soit le traitement hypophysaire.

(1) Centre d'Alevinage et de Recherches Piscicoles (C.A.R.P.) de Brenne - BENAVANT 36300 LE BLANC

(2) Rapportées d'URSS. 


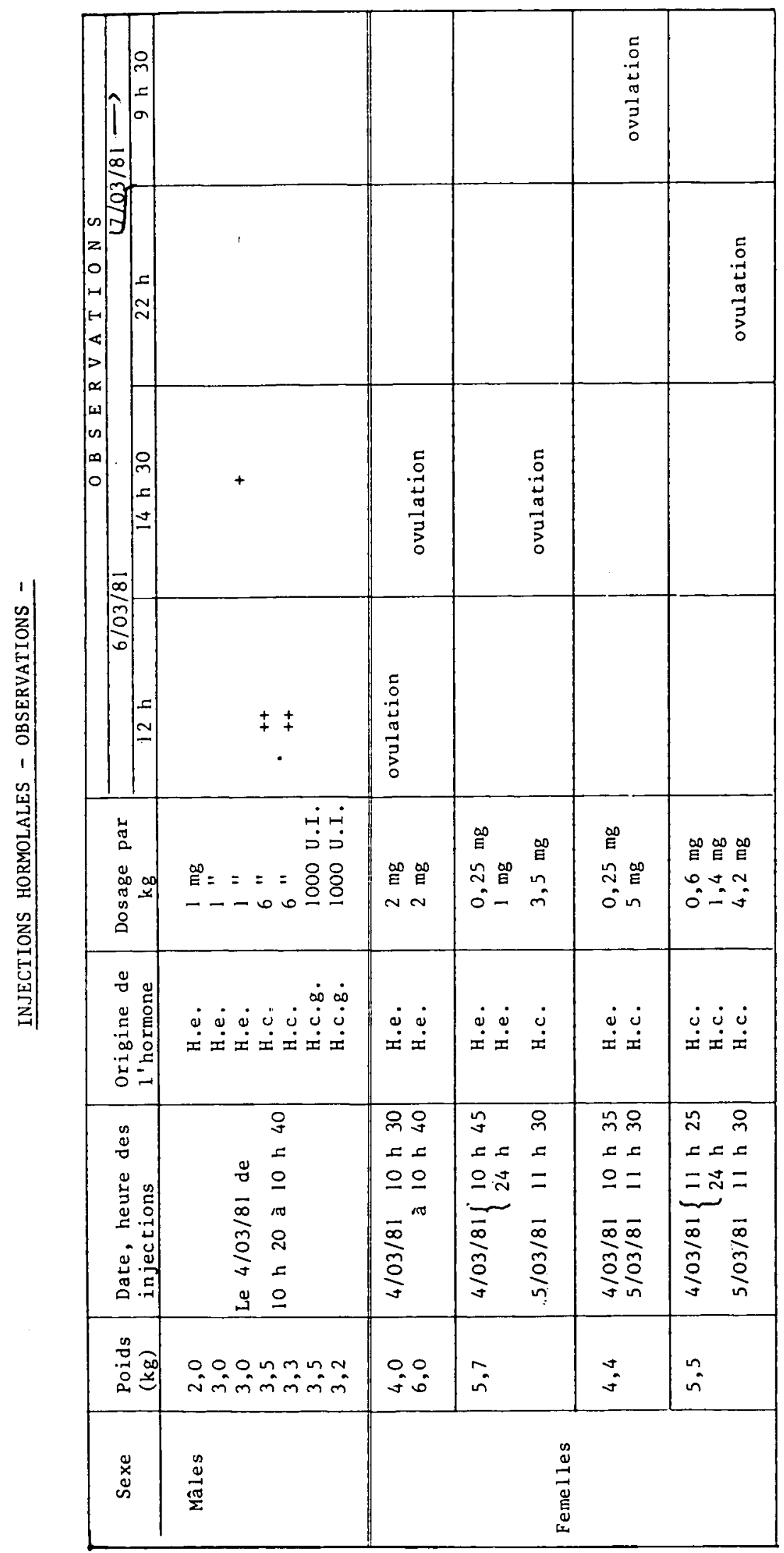


Les mâles: Seuls 3 sur 7 ont donné du sperme par pression abdominale; et encore faut-il remarquer que les 2 poissons qui en ont donné le plus sont ceux ayant reçu une injection d'hypophyse de carpe. Néanmoins, il faut bien se garder de conclure hâtivement quant à la supériorité absolue d'un traitement par rapport à un autre surtout dans le cas des mâles. La méthode utilisée, relativement imprécise, par manque de connaissance interdit toute généralisation. Pour les mâles enfin, aucun témoin avec seulement une injection de solution saline n'a été retenu eu égard au faible nombre d'animaux.

\subsection{Prélèvements des gamètes}

Après chaque observation jugée favorable sur une femelle du sperme des 3 mâles était récolté.

Des mesures volumétriques n'ont pas été faites. Ensuite chaque femelle était anesthésiée selon la même méthode que pour les biopsies. Elle était rincée, essuyée et maintenue verticalement la tête en haut. On pratiquait alors une incision de l'abdomen depuis quelques centimètres au-dessus de l'orifice génital en remontant vers la tête et ceci sur 25 à $35 \mathrm{~cm}$. Les ovules recueillis dans une cuvette étaient alors pesés.

La quantité moyenne par individu ainsi obtenue a été de $530 \mathrm{~g} \pm 115$ ce qui correspond à un rapport gonado-somatique (1) de 0,12 $\pm 0,02$. Le diamètre des ovules était voisin ou légèrement supérieur à $2,5 \mathrm{~mm}$.

\subsection{Fécondation}

Pour chaque fécondation nous avons utilisé un mélange du sperme des 3 mâles à raison de $10 \mathrm{~cm}^{3}$ par kilogramme d'ovules, ce mélange étant ensuite dilué à raison de 1/200 (WILLIOT, CHARLON, 1978). Cette dilution est nécessaire pour éviter les phénomènes de polyspermies, les ovules d'esturgeons possédant plusieurs micropyles. Ces normes sont celles utilisées par les soviétiques, sans que nous sachions si elles sont parfaitement adaptées à l'esturgeon sibérien.

Pratiquement, deux diluants ont été testés:

- de l'eau:

- solution aqueuse à $3 \%$ d'urée et $4 \%$ de chlorure de sodium, cette formule étant connue pour son pouvoir d'activateur de la fécondation chez les cyprinidés.

II n'est pas certain que ćette formulation soit la meilleure pour $A$. baeri.

Le mélange (ovule + solution fécondante) a été brassé manuellement durant environ 5 minutes puis rincé plusieurs fois.

\subsection{Traitement des ceufs}

Pour s'affranchir de l'adhérence des œufs qui empêcherait un bon développement en incubateur il est nécessaire de les traiter. Plusieurs solutions ont été testées:

- suspension aqueuse d'argile avec brassage durant 15 à 20 minutes:

- lait dilué dans l'eau dans un rapport 1/5, brassage pendant 1 heure;

- solution aqueuse d'acide tanique à $1 \mathrm{~g} / \mathrm{l}$ pendant 20 secondes.

Après chaque traitement, les œufs ont été soigneusement rincés. Les 3 traitements se sont avérés efficaces pour éviter l'agglomération des œufs. II n'a pas été possible de suivre précisément la survie embryonnaire et l'influence de ces traitements reste à préciser.

Cependant, d'un point de vue pratique et dans des conditions manuelles de traitement, le lait entraîne une manipulation longue, alors que le tanin est très délicat d'utilisation (rapidité d'action).

\section{INCUBATION}

L'incubation a eu lieu dans des bouteilles de Zoug du 6 au 22 mars, soit 16 jours. Du 6 au 18, la température a dû être maintenue entre 8 et $11^{\circ} \mathrm{C}$, la grosse majorité des bouteilles étant alors utilisée pour l'incubation d'œufs de brochet. Durant les trois ou quatre derniers jours la température a été portée entre 14 et $16^{\circ} \mathrm{C}$.

Seuls certains stades très différenciés de l'embryogenèse ont pu être observés.

(1) R.G.S. = (poids vif - poids ovules) / poids vif 
Ainsi par exemple le début du stade gastrula (apparition du bouchon vitellin au pôle végétatif, stade 15-16 d'après DETLAF et GINSBOURG, 1969) correspond à une fourchette de 750 à $850^{\circ} \mathrm{C}$ - heure - soit environ 4 jours dans les conditions d'essai. Les premières éclosions sont intervenues après 16 jours, șoit $4000^{\circ} \mathrm{C} \times$ heure.

En fait, les éclosions se sont étalées sur plus de 48 heures. Durant la durée de l'incubation un traitement au vert malachite a eu lieu chaque jour.

Pour les 5 femelles, quels que soient le procédé de fécondation et le traitement des œufs, l'embryogenèse a atteint le stade gastrula.

Aucun des œufs venant de 2 femelles ayant reçu un traitement hypophysaire différent n'est parvenu à l'éclosion.

\section{TRAITEMENT DES GÉNITEURS}

A l'issue de ces essais, les mâles ont été relâchés directement en étang. Quatre des cinq femelles ont été recousues puis remises en étang.

\section{DISCUSSION}

Les travaux sur la reproduction de l'esturgeon sibérien répondent à deux objectifs simultanés:

- acquérir une expérience sur les acipenséridés

- augmenter notre stock expérimental.

La difficulté était de choisir les.moyens à mettre en œuvre pour atteindre ces buts. Pour chaque phase, sauf une, nous avons pris comme référence la technique développée par les soviétiques sur les grands migrateurs sans pour autant savoir si elle convient à l'esturgeon sibérien. Afin de résoudre cette question il eut été souhaitable que le protocole expérimental soit plus rigoureux. Cela était impossible pour les trois raisons suivantes:

- manque d'expérience et de connaissance sur la physiologie de la reproduction de cette espèce. Ainsi nous n'avons aucune certitude que les animaux sur lesquels nous avons travaillé se trouvaient exactement au même stade de la gamétogenèse; il est donc impossible de conclure nettement sur les divers traitements retenus.

- la remarque précédente est accentuée par le faible nombre d'animaux

- enfin les conditions de travail ne nous ont pas permis d'effectuer toutes les observations qu'il eut été souhaitable de faire.

En dépit de ces remarques, les résultats obtenus font apparaître quelques aspects intéressants.

Traitement hypophysaire: même si cela n'est peut-être pas la meilleure solution, il est possible d'utiliser de l'hypophyse de carpe pour stimuler la maturation. Donc à court et moyen termes ceci nous permettra de continuer ces travaux bien que nous n'ayons presque plus d'hypophyse d'esturgeon. Les doses retenues ainsi que leur fractionnement devront faire l'objet de recherches ultérieures. Il est vraisemblable par exemple que les doses ( $6 \mathrm{mg} / \mathrm{kg}$ pour les 2 sexes) puissent être diminuées notamment pour les mâles.

Traitement des œufs fécondés : dans le cadre d'un traitement manuel, la suspension d'argile s'avère plus facile à mettre en œuvre car plus rapide. Cependant dans l'hypothèse de traitement automatique l'utilisation du lait pourrait peut-être s'avérer intéressante.

Incubation : pour cette phase nous n'avons pas pu comparer le type d'incubation soviétique avec les bouteilles de Zoug. II sera nécessaire utérieurement de faire cette comparaison.

Enfin la suite directe de ce travail consistera surtout à élever les larves obtenues et à suivre la survie et l'évolution de la gamétogenèse des animaux que nous avons utilisés.

\section{REMERCIEMENTS}

Ces travaux n'ont été possibles que grâce à l'utilisation d'une partie des installations de la pisciculture de I'INRA à DONZACQ (40) puis celles du Centre d'Alevinage et de Recherches piscicoles (C.A.R.P.) de Brenne. Nous tenons à remercier tout particulièrement pour leur aide et leur bienveillance Monsieur et Madame SZABO. Messieurs RETAUD et COUTURIER. 


\section{BIBLIOGRAPHIE}

BARRUCAND M., FERLIN P., LAMARQUE P., SABAUT J.J. Alimentation artificielle de l'esturgeon Acipenser bạeri. Symposium FAO sur la nutrition des poissons et la technologie de leurs aliments artificiels, Hambourg, 20-23 juin 1978.

BRETON B., FOSTIER A., JALABERT B., WEIL C. Contrôle du cycle reproducteur des poissons dans : R. BILLARD, La pisciculture en étang, INRA, 1980

C.T.G.R.E.F. Utilisation d'eaux réchauffées dans des condenseurs. Expérimentation menée à la centrale thermo-électrique de Bordeaux-Ambès, rapport final semestriel no6, juin 1979.

C.T.G.R.E.F. Pisciculture de la carpe commune Cyprinus carpio et reproduction artificielle de l'esturgeon Acipenser ruthenus, rapport mission en Hongrie, mai 1980.

DETLAF T.A. et GINSBOURG A.S. Développement des Acipenséridés. Maturation des œufs, fécondation et embryogenèse. Éditions Sciences, Moscou, 1969.

KHAKIMOULLIN A.A. Durée de maturation de l'esturgeon sibérien après injection , hypophysaire. Économie des pêches no 8, 1979. Éd. : Industries alimentaires et légères, Économie des Pêches, URSS.

MARCEL J. Préparation et utilisation de broyats hypophysaires pour l'induction de la reproduction des poissons dans : R. BILLARD, La pisciculture en étang, INRA, 1980.

WILLIOT P. et CHARLON N. Rapport sur l'élevage des esturgeons en URSS. Ministère de I'Agriculture, CTGREF - INRA, janvier 1978. 